\title{
Hydrothermal time germination models for radiata pine (Pinus radiata D. Don)
}

\author{
M. Bloomberg ${ }^{1,2 *}$, J.R. Sedcole ${ }^{1}$, E.G. Mason ${ }^{2}$ and G. Buchan ${ }^{1}$ \\ ${ }^{1}$ Agriculture and Life Sciences Division, Lincoln University, Canterbury, New Zealand; ${ }^{2}$ School of Forestry, \\ University of Canterbury, Christchurch, New Zealand
}

\begin{abstract}
The objective of this study was to fit a hydrothermal germination model to germination data for a seedlot of radiata pine (Pinus radiata D. Don). Seeds were incubated for $50 \mathrm{~d}$ at constant temperatures and water potentials $\left(T=12.5-32.5^{\circ} \mathrm{C}, \Psi=0\right.$ to $\left.-1.2 \mathrm{MPa}\right)$. Most seeds completed germination within $50 \mathrm{~d}$, but for low $\Psi$ and/or non-optimal temperatures $\left(T<17.5^{\circ} \mathrm{C}\right.$, $T>25^{\circ} \mathrm{C}$ ) many seeds did not complete germination. In general, germination data conformed to the hydrothermal model. Departures from the model were encountered for slow-germinating seeds at suboptimal temperatures $\left(T \leq 20^{\circ} \mathrm{C}\right)$. To account for these departures, two alternative hydrothermal models were fitted with an additional term for an upwards shift in seed base water potential with increasing time to germination. The alternative models more correctly predicted germination time than the original model. Similarly, reduced percentage germination at supra-optimal temperatures $\left(T>20^{\circ} \mathrm{C}\right)$ was explained by including a term in the hydrothermal model which shifted the base water potential of seeds upwards towards zero, which in turn reduced the predicted rate that hydrothermal time would be accumulated by seeds. The rate of this upwards shift in base water potential was dependent on time to complete germination and ambient water potential as well as supra-optimal temperature.
\end{abstract}

Keywords: hydro-time, Monterey pine, seeds, thermal time, water relations

\section{Introduction}

The objective of this study was to fit a hydrothermal germination model to germination data for a seedlot of radiata pine (Pinus radiata D. Don), an important

*Correspondence

Email: markbloomberg@xtra.co.nz plantation forest species. Hydrothermal germination models offer a useful way to understand why and how seed germination varies under different seedbed conditions, and between different seed populations. In hydrothermal models, germination rate and percentage germination are explained by seed population characteristics and by the water potential $(\Psi)$ and temperature $(T)$ of the seed's environment. The hydrothermal germination model was first proposed by Gummerson (1986) and can be specified mathematically as:

$$
\theta_{H T}=\left[\Psi-\Psi_{b}(\mathrm{G})\right]\left(T-T_{b}\right) t(\mathrm{G})
$$

where $\theta_{H T}$ is a hydrothermal time constant that has a unique value for the seed population $\left(\mathrm{MPa}{ }^{\circ} \mathrm{Cd}\right)$, $\Psi_{b}(\mathrm{G})$ is the base water potential for the Gth percentile of the seed population ( $\mathrm{MPa})$, and varies according to a frequency distribution, $T_{b}$ is the base temperature for seed germination $\left({ }^{\circ} \mathrm{C}\right)$ and $t(\mathrm{G})$ is the time for the Gth percentile to germinate $(d)$.

The model predicts that under constant conditions of $T$ and $\Psi$, the Gth seed in the population will germinate when the seed has accumulated hydrothermal time [calculated from the right-hand side of equation (1)] equal to the population hydrothermal time constant $\theta_{H T}$.

A standard assumption in hydrothermal time models is that $\Psi_{b}(\mathrm{G})$ varies within the population but $T_{b}$ has a unique value. Hence all seeds in the population will accumulate the same thermal time under any temperature regime in the seed's environment. In contrast, because $\Psi_{b}(\mathrm{G})$ is different for each seed percentile, each percentile will accumulate different amounts of 'hydro-time' within a specific time period under any moisture regime applied to the whole seed population. Therefore it is the variation in $\Psi_{b}(G)$ that results in a spread of seed germination times within the population (Bradford, 2002; Finch-Savage, 2004). Hydrothermal germination models conventionally assume that $\Psi_{b}(\mathrm{G})$ is normally distributed, so the median base water potential [ $\left.\Psi_{b}(50)\right]$ will equal the mean $\Psi_{b}(\mathrm{G})$ for the seed population. 


\section{Modelling departures from the conventional hydrothermal germination model}

Departures of actual seed germination behaviour at suboptimal temperatures from the conventional hydrothermal model are frequently reported in the literature (Dahal and Bradford, 1994; Phelps and Finch-Savage, 1997; Kebreab and Murdoch, 1999; Grundy et al., 2000). These departures may necessitate changing the assumptions of the model, for example by varying the value of $\Psi_{b}(\mathrm{G})$ depending on ambient water potential (Dahal and Bradford, 1994).

In addition, the hydrothermal model specified by equation (1) implies that seed germination rate (GR) will increase indefinitely with increasing $T$, i.e. if the term $\left(T-T_{b}\right)$ becomes larger and if $\theta_{H T}$ is a constant, then $t(G)$ must become smaller. In reality, as temperature increases both GR and germination percentage reach a maximum at the optimum temperature for germination $\left(T_{o}\right)$ and then decline to zero at a ceiling temperature $\left(T_{c}\right)$. Alvarado and Bradford (2002) and Rowse and Finch-Savage (2003) have proposed hydrothermal models that account for this decline by adjusting the base water potential of seeds upwards towards zero at supra-optimal temperatures. The Alvarado and Bradford (2002) and Rowse and Finch-Savage (2003) models are referred to in this paper as the AB and RFS models, respectively.

In both the $\mathrm{AB}$ and RFS models, the upwards adjustment in base water potential reduces the rate of hydro-time accumulation by the seed, and so it takes longer to accumulate hydrothermal time equal to $\theta_{H T}$, which it must do before it can complete germination. The AB model assumes that the maximum rate of thermal time accumulation occurs at $T_{o}$, and that supra-optimal temperatures do not contribute additional hydrothermal time, i.e. for $T>T_{o}$, thermal time $=\left(T_{o}-T_{b}\right) t(G)$. It also assumes that the upwards adjustment in seed base water potential scales linearly with supra-optimal temperature, as shown by equation (2):

$$
\Psi_{b}(\mathrm{G})_{\text {sup }}=\Psi_{b}(\mathrm{G})_{o}+k\left(T-T_{o}\right) \text { when } T>T_{o}
$$

where $\Psi_{b}(\mathrm{G})_{\text {sup }}$ is the adjusted seed base water potential at supra-optimal temperatures, and $\Psi_{b}(\mathrm{G})_{0}$ is the base water potential of seeds which applies at $T=T_{o}$. The effects of these two assumptions are that: (1) the relationship between GR and temperature follows a 'broken-stick' pattern, with a linear upwards trend in GR from $T_{b}$ to $T_{o}$ followed by an abrupt transition to a linear decline in GR down to $T_{c}$; and (2) the peak of the 'broken stick' occurs at the same temperature $\left(T_{0}\right)$ for all seed percentiles (Alvarado and Bradford, 2002).

The RFS model differs importantly from the $A B$ model in that there is no maximum temperature for calculating thermal time, i.e thermal time accumulates increasingly rapidly with increasing temperature above $T_{o}$. This means that predicted GR continues to increase above the temperature at which seed base water potential begins to increase as a function of supra-optimal temperature, because the extra thermal time accumulated at higher temperatures counteracts the effect of an upwards adjustment in $\Psi_{b}(\mathrm{G})_{\text {sup }}$ towards zero (Rowse and Finch-Savage, 2003). However, the upwards-adjusting effect of temperature on $\Psi_{b}(\mathrm{G})_{\text {sup }}$ occurs at a faster rate per unit of temperature than thermal time accumulation, and so there is an inflexion point where GR begins to decline. These assumptions mean that for the RFS model, 'deviation temperature' or $T_{d}$ (the temperature threshold where $\Psi_{b}(\mathrm{G})_{\text {sup }}$ begins to increase as a function of temperature) is substituted in equation (2) for the actual optimum temperature $T_{o}$, where GR is at a peak. The assumptions of the RFS model also mean that: (1) the plot of predicted GR versus $T$ has a broad, rounded peak (rather than a sharp 'broken-stick' profile); and (2) $T_{o}$ occurs at a higher temperature for the lower percentile than for higher percentile seeds (Rowse \& FinchSavage, 2003). The next sections describe a germination study using seed from radiata pine. The aim of the study was to test the general hydrothermal model [equation (1)] for germination at suboptimal temperatures, and the AB and RFS models [equation (2)] for germination at supra-optimal temperatures.

\section{Methods}

A commercial radiata pine seedlot (seedlot no. 075/780 supplied by Proseed NZ Ltd, Amberley, New Zealand) was incubated for $50 \mathrm{~d}$ in incubators at constant temperatures from 12.5 to $32.5^{\circ} \mathrm{C}$, and at constant water potentials from 0 to $-1.2 \mathrm{MPa}$. Pilot experiments with seedlot no. 075/780 suggested that optimum germination was obtained at temperatures of $20-22^{\circ} \mathrm{C}$ and water potential $=0 \mathrm{MPa}$ (cf. ISTA, 2003) and that base temperature and mean base water potential for germination were approximately $10^{\circ} \mathrm{C}$ and $-1.5 \mathrm{MPa}$, respectively.

For each combination of water potential and temperature there were four replicates, each comprising a $750 \mathrm{ml}$ plastic snap-top tray with 25 seeds on hydrated Whatman No. 2 filter papers. Before placement in the trays, filter papers were soaked in trays containing an osmotic solution of the desired water potential for $48 \mathrm{~h}$. The filter papers were then placed on a $5 \mathrm{~mm}$ thick glass plate in the trays which were filled with freshly mixed osmotic solution until the solution just made contact with the top of the filter paper. The glass plates were of smaller dimensions than the plastic trays, which meant there was a reservoir of osmotic solution surrounding each plate. This was intended to further buffer the filter paper from changes in water potential due to evaporation of 
water. Osmotic solutions of water potential $=0,-0.3$, $-0.6,-0.9$ and $-1.2 \mathrm{MPa}$ were made up using polyethylene glycol 8000 (PEG 8000) according to equation (6) in Hardegree and Emmerich (1990). The osmotic solution with $0 \mathrm{MPa}$ was reverse osmosis water with no PEG 8000 added.

Seeds were screened to remove any obvious empty or broken seeds and then lightly dusted with fungicide (Thiram) before being carefully placed on to the germination medium. Seeds were expected to have better than 95\% germinability, based on seed-testing results provided by Proseed NZ Ltd. Radiata pine seed has no stratification-sensitive seed dormancy (Rimbawanto et al., 1988), therefore seeds received no stratification or other pre-treatment prior to sowing (ISTA, 2003). Once the seeds were placed in the germination trays, the tops of the trays were sealed with a sheet of cling-wrap and the plastic snap-top lid was firmly placed on the top of the tray. Trays were then placed in eight incubators at constant temperatures of $12.5,15,17.5,20,22.5,25,27.5$ and $32.5^{\circ} \mathrm{C}$ respectively. All water potential treatments had four replicates in each incubator, except for $-1.2 \mathrm{MPa}$ which was only replicated for $T=17.5$ and $20^{\circ} \mathrm{C}$. Within each incubator, trays were randomly placed and positions were rotated systematically every $3 \mathrm{~d}$ to mitigate any small spatial variations in air temperature due to inefficient air circulation. Temperatures were monitored continuously within the incubators using either Tiny-Tag temperature loggers (Gemini Data Loggers, Chichester, UK) or temperature probes interfaced with a HOBO data logger (Onset Computer Corporation, Bourne, Massachusetts, USA). No consequential deviations from target incubator temperatures occurred during the experiment. Water potentials of the germination media were monitored by measuring samples of the osmotic solutions in the trays twice weekly, using a Wescor 5520 vapour pressure osmometer (Wescor Inc., Logan, Utah, USA). Where osmotic potentials deviated from a specified treatment level by $>0.05 \mathrm{MPa}$, that osmotic solution was discarded and replaced with fresh solution.

Germination counts were continued for a period of $50 \mathrm{~d}$. Seeds were inspected twice daily for the first $9 \mathrm{~d}$ of the incubation period, and thereafter daily until $27 \mathrm{~d}$, after which seeds were inspected every $2 \mathrm{~d}$. Seeds were considered to have germinated when the radicle protruded more than $2 \mathrm{~mm}$ from the seed coat. Germinated seeds were counted and discarded. Seed counting required the trays to be opened, which also served to aerate them.

\section{Assessment of dormancy}

Most seeds germinated within $50 \mathrm{~d}$ for the nearoptimum treatments. However, for treatments with non-optimal temperatures and/or low water potentials, many seeds remained ungerminated after $50 \mathrm{~d}$. This may have been due to the induction of secondary dormancy. The extent of this possible dormancy was tested after $50 \mathrm{~d}$ by placing all remaining ungerminated seeds in trays with water $(\Psi=0 \mathrm{MPa})$ and incubating them at optimum temperature $\left(20^{\circ} \mathrm{C}\right)$ for a further $75 \mathrm{~d}$. Counts of germination were taken at $15 \mathrm{~d}$ intervals and, as with the main experiment, germinated seeds were counted and discarded, as were any seeds that were clearly dead (squash test showed loss of structure). Seeds that germinated under ideal conditions within $75 \mathrm{~d}$ were considered non-dormant. Seeds that had not germinated by $75 \mathrm{~d}$ were considered completely dormant, although they may simply have been non-viable.

\section{Data analysis}

\section{Germination rate}

The germination rate (GR) of a seed is defined as the inverse of the time taken by the seed to germinate, i.e. $1 / \mathrm{GR}=t(\mathrm{G})$. By plotting GR versus $\Psi$ and $T$, data can be checked for their conformity to the hydrothermal model, i.e. for all percentiles: (1) GR versus T should be linear for $T<T_{o}$ and converge to a single base temperature $T_{b}$; and (2) GR should increase linearly as water potential increases from $\Psi=\Psi_{b}$ (where $\mathrm{GR}=0$ ) to $\Psi=0$ (where GR reaches its maximum value). In addition, for GR versus $\Psi$, the trend lines for the different percentiles should be parallel, with each percentile having a common slope but a differing intercept $\left[=\Psi_{b}(\mathrm{G})\right]$ with the $x$-axis (Bradford, 2002).

\section{Estimating hydrothermal germination model parameters}

Germination data for the four 25-seed replicates for each temperature/water potential treatment were amalgamated into one set of data (i.e. 100 seeds). Within each treatment, seeds were allocated a percentile number $(G)$, beginning with 1 for the fastest seed to germinate, 2 for the second-fastest and so on. It was assumed that all seeds were potentially germinable, and so the maximum percentile number for any treatment was 100. In order to estimate parameter values for equation (1), it was transformed as follows (Gummerson, 1986):

$$
\operatorname{Probit}(\mathrm{G})=\left[\Psi-\Psi_{b}(50)-\left\{\theta_{H T} /\left(T-T_{b}\right) t(\mathrm{G})\right\}\right] / \sigma_{\Psi b}
$$

where Probit $(G)$ is the probit function which calculates the standard normal deviate $(z)$ for a specified cumulative probability $(=\mathrm{G})$ in a normally distributed population, $\Psi_{b}(50)$ is the mean $\Psi_{b}(\mathrm{G})$ and $\sigma_{\Psi b}$ is the standard deviation of $\Psi_{b}(\mathrm{G})$ for the seed population. 
For the germination data at suboptimal temperatures $\left(12.5-20^{\circ} \mathrm{C}\right)$, the Probit $(\mathrm{G})$ statistics for all seeds were calculated, then equation (3) was fitted to the germination data using the 'nls' function in programme $\mathrm{R}$ ( $\mathrm{R}$ Development Core Team, 2007). This function simultaneously estimated $\theta_{H T}, \Psi_{b}(50), T_{b}$ and $\sigma_{\Psi b}$ using an iterative least-squares procedure.

\section{Checking the hydrothermal germination model}

Departures from the conventional hydrothermal model may arise when seed base water potential $\left[\Psi_{b}(\mathrm{G})\right]$ varies from the assumptions that its values are: (1) independent of germination conditions; and (2) normally distributed around a mean $\Psi_{b}(50)$ (Dahal and Bradford, 1994; Alvarado and Bradford, 2002; Rowse and Finch-Savage, 2003). For any mathematical model, model goodness of fit to the data can be checked using the raw residuals (= observed values for the response variable - predicted values) (Crawley, 2002). Systematic biases or trends in the raw residuals for seed base water potential would indicate that assumptions 1 and 2 above were not valid. The predicted value for seed base water potential is easy to calculate, because if $\Psi_{b}(\mathrm{G})$ is normally distributed around a mean $\left[\Psi_{b}(50)\right]$ then it is axiomatic that the predicted value $\Psi_{b}(\mathrm{G})_{\text {pred }}$ will be:

$$
\Psi_{b}(\mathrm{G})_{\text {pred }}=\Psi_{b}(50)+\operatorname{Probit}(\mathrm{G}) \sigma_{\Psi b}
$$

This provides a fixed value for $\Psi_{b}(\mathrm{G})$ across all environments. However, determining an observed value is less obvious because values of $\Psi_{b}(\mathrm{G})$ are not directly observed. To overcome this problem, equation (1) can be rearranged to give:

$$
\Psi_{b}(\mathrm{G})^{\prime}=\Psi-\left\{\theta_{\mathrm{HT}} /\left(T-T_{b}\right) t(\mathrm{G})\right\}
$$

By substituting model parameters estimated using equation (3) and observed values for $t(G), T$ and $\Psi$ into the right-hand side of equation (5), the calculated value of the left-hand side of equation (5) is a virtual 'observed value' for seed base water potential. Unlike $\Psi_{b}(\mathrm{G})_{\text {pred }}$, which has a unique constant value for each seed percentile, $\Psi_{b}(G)^{\prime}$ can vary between different water potential and temperature treatments.

The model raw residuals [denoted by $\Delta \Psi_{b}$, where $\left.\Delta \Psi_{b}=\Delta \Psi_{b}(\mathrm{G})^{\prime}-\Delta \Psi_{b}(\mathrm{G})_{\text {pred }}\right]$ can therefore reveal whether seed base water potential varies systematically with water potential and temperature treatments, as follows:

(1) If seed base water potential values of imbibed seeds change upwards or downwards in response to germination conditions, as suggested by Dahal and Bradford (1994), then raw residuals $\left(\Delta \Psi_{b}\right)$ should show a systematic trend upwards or downwards with respect to those germination conditions.

(2) For seeds incubated at supra-optimal temperatures, raw residuals $\left(\Delta \Psi_{b}\right)$ can be calculated from equation (2), by assuming that: (i) seed base water potential at $T_{o},\left[\Psi_{b}(\mathrm{G})_{o}\right]$ is equal to $\Psi_{b}(\mathrm{G})_{\text {pred }}$ [equation (4)]; and (ii) $\Psi_{b}(\mathrm{G})^{\prime}$ [equation (5)] is equal to $\Psi_{b}(\mathrm{G})_{\text {sup }}$. Then substituting $\Psi_{b}(\mathrm{G})^{\prime}$ and $\Psi_{b}(\mathrm{G})_{\text {pred }}$ into equation (2) gives:

$$
\Psi_{b}(\mathrm{G})^{\prime}=\Psi_{b}(\mathrm{G})_{\text {pred }}+k\left\{T-T_{o}\right)
$$

$\Delta \Psi_{b}$ should therefore be linearly related to supra-optimal temperature, because if $\Delta \Psi_{b}=$ $\Psi_{b}(\mathrm{G})^{\prime}-\Psi_{b}(\mathrm{G})_{\text {pred }}$, then $\Delta \Psi_{b}=k\left\{T-T_{o}\right)$. Note that for the RFS model, $\Psi_{b}(\mathrm{G})_{d}$ and $T_{d}$ (where $d=$ deviation temperature) are substituted for $\Psi_{b}(\mathrm{G})_{o}$ and $T_{o}$.

The assumption that seed base water potential is normally distributed can be checked in similar fashion. If the frequency distribution of $\Psi_{b}(\mathrm{G})$ is normal, then at suboptimal temperatures $\Psi_{b}(\mathrm{G})^{\prime}$ is expected to equal $\Psi_{b}(\mathrm{G})_{\text {pred }}$ and $\Delta \Psi_{b}$ should be distributed around a mean of zero with no bias in the distribution of its values with respect to seed percentile. Conversely, if the frequency distribution of $\Psi_{b}(\mathrm{G})$ is non-normal, then at suboptimal temperatures $\Delta \Psi_{b}$ should exhibit a systematic bias in the distribution of its values around its mean, with respect to seed percentile.

$\Delta \Psi_{b}$ for seeds incubated at suboptimal temperature was plotted against seed percentile to check the assumption that seed base water potential was normally distributed. $\Delta \Psi_{b}$ for seeds incubated at supra-optimal temperatures was plotted against temperature, to verify whether seed base water potential increased towards zero with increasing supra-optimal temperatures, as proposed by the $\mathrm{AB}$ and RFS models.

\section{Results}

\section{Germination rate}

Plots of seed GR versus temperature (for optimum $\Psi=0 \mathrm{MPa}$ ) and water potential (for $T=20^{\circ} \mathrm{C}$ ) are shown in Fig. 1a and b. Rates are plotted for the 10th, 30th, 50th, 70th and 90th percentiles, to show trends in data across the entire seed population. In general, GRs of the radiata pine seeds at temperatures less than or equal to $20^{\circ} \mathrm{C}$ conform to the assumptions of the hydrothermal model, i.e. for all percentiles: (1) GR versus $T$ appears to be linear converging to a single base temperature $T_{b}$; and (2) GR versus $\Psi$ appears to be linear with parallel trend lines for each percentile and with each percentile having a different intercept $\left[=\Psi_{b}(\mathrm{G})\right]$ with the $x$-axis. 

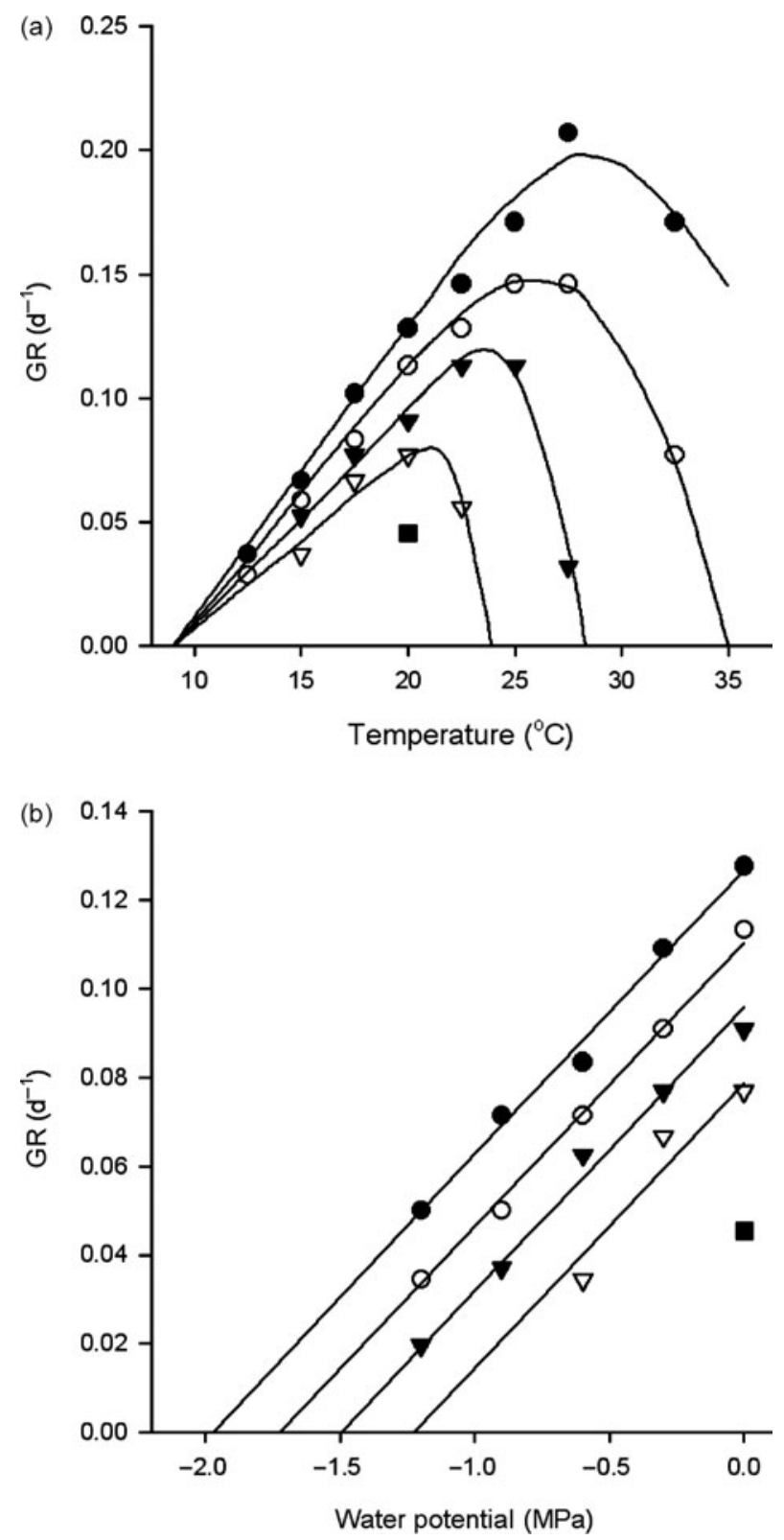

Figure 1. Germination rates (GR) for 10th to 90th percentiles versus: (a) temperature when $\Psi=0 \mathrm{MPa}$; (b) water potential when $T=20^{\circ} \mathrm{C}$. Percentile symbols: $0=10$ th, $O=30$ th, $\boldsymbol{\nabla}=50$ th,$\nabla=70$ th, $\boldsymbol{\square}=90$ th. The solid lines are drawn freehand to illustrate trends in GR versus temperature and water potential. Lines in (a) are constrained to pass through a single $T_{b}$ of $9.0^{\circ} \mathrm{C}$. Lines in (b) are constrained to a common slope. There is no line drawn for the 90th percentile because $90 \%$ germination was only achieved for $20^{\circ} \mathrm{C}$ and $0 \mathrm{MPa}$.

For temperatures greater than $20^{\circ} \mathrm{C}$, the rate of increase in GR with temperature appears to decline non-linearly until $T_{o}$ (the optimum temperature where GR is at a maximum). Beyond $T_{o}$, GR declines (quite rapidly for the higher percentiles) to zero at the ceiling temperature $T_{c}$. However, the values of $T_{o}$ and $T_{c}$ are different for each percentile of the seed population. $T_{o}$ and $T_{c}$ are typically highest for the fastest germinating seeds (i.e. the lowest percentiles in the population). Figure 1a shows that GR for the 10th percentile only just began to decline at $32.5^{\circ} \mathrm{C}$ and it is difficult to estimate $T_{c}$ for this percentile by extrapolation. These trends strongly suggest that germination of radiata pine at supra-optimal temperatures conforms more closely to the RFS model than to the AB model, because there is a broad range of optimum temperatures which vary between different seed percentiles, rather than a unique value for $T_{o}$ which applies to all percentiles. They also suggest that the deviation temperature $\left(T_{d}\right)$ where $\Psi_{b}$ begins to decline is $\approx 20^{\circ} \mathrm{C}$.

\section{Hydrothermal models at suboptimal temperatures}

Estimated hydrothermal model parameters for equation (3) are shown in Table 1 (Model 1). These were used to calculate $\Delta \Psi_{b}\left[=\Psi_{b}(\mathrm{G})^{\prime}-\Psi_{b}(\mathrm{G})_{\text {pred }}\right]$, equations (4) and (5), which was then plotted against seed percentile, to verify whether the data conformed to the assumption that the seed base water potential is normally distributed [equation (1)]. Figure 2a $\left(\Delta \Psi_{b}\right.$ versus seed percentile) shows that although the distribution of $\Delta \Psi_{b}$ is biased, biases in distribution are not consistent with respect to percentile for seeds incubated at different temperatures or water potentials. This suggests that biases were not caused by an underlying non-normal distribution of $\Psi_{b}(\mathrm{G})$ in the seed population. However, biases in $\Delta \Psi_{b}$ did appear to be related to the germination time for the seed, suggesting that seed base water potentials changed during the germination time course (Fig. 2b). For seeds that took less than $25 \mathrm{~d}$ to germinate, $\Delta \Psi_{b}$ was more or less uniformly distributed around 0 , i.e. seed base water potential did not change during this time. For seeds that took longer than $25 \mathrm{~d}$ to germinate, $\Delta \Psi_{b}$ increased linearly with respect to germination time, which meant they germinated even slower. Figure $2 b$ also shows that the relationship of $\Delta \Psi_{b}$ versus time was consistent for all temperatures but varied with $\Psi$. For the driest treatment $(-1.2 \mathrm{MPa})$ the relationship became weak. To account for the relationship between $\Delta \Psi_{b}$ and time, two alternative hydrothermal models

Table 1. Model parameters and Akaike Information Criterion (AIC) values for suboptimal hydrothermal models

\begin{tabular}{lccc}
\hline & Model 1 & $\begin{array}{c}\text { Model 2 } \\
\text { Time-adjusted }\end{array}$ & $\begin{array}{c}\text { Model 3 } \\
\text { PTI adjusted }\end{array}$ \\
Parameter & Unadjusted & $\Psi_{b}$ [equation (7)] & $\Psi_{b}$ [equation (8)] \\
\hline$\theta_{H T}\left(\mathrm{MPa}{ }^{\circ} \mathrm{C}\right.$ d) & 149 & 168 & 176 \\
$T_{b}\left({ }^{\circ} \mathrm{C}\right)$ & 9.7 & 9.4 & 9.0 \\
$\Psi_{b}(50)(\mathrm{MPa})$ & -1.38 & -1.50 & -1.53 \\
$\sigma_{\Psi b}(\mathrm{MPa})$ & 0.48 & 0.47 & 0.42 \\
$K$ & - & $1.17 \times 10^{-2}$ & $1.30 \times 10^{-2}$ \\
$\mathrm{AIC}$ & 67.6 & -136 & -449 \\
\hline
\end{tabular}




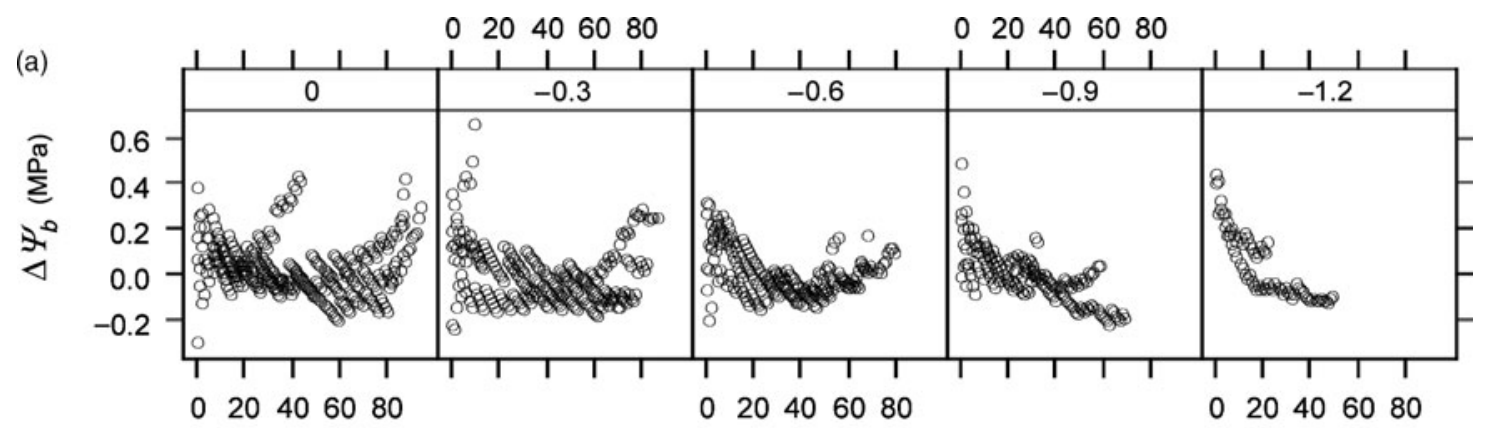

Germination percentile
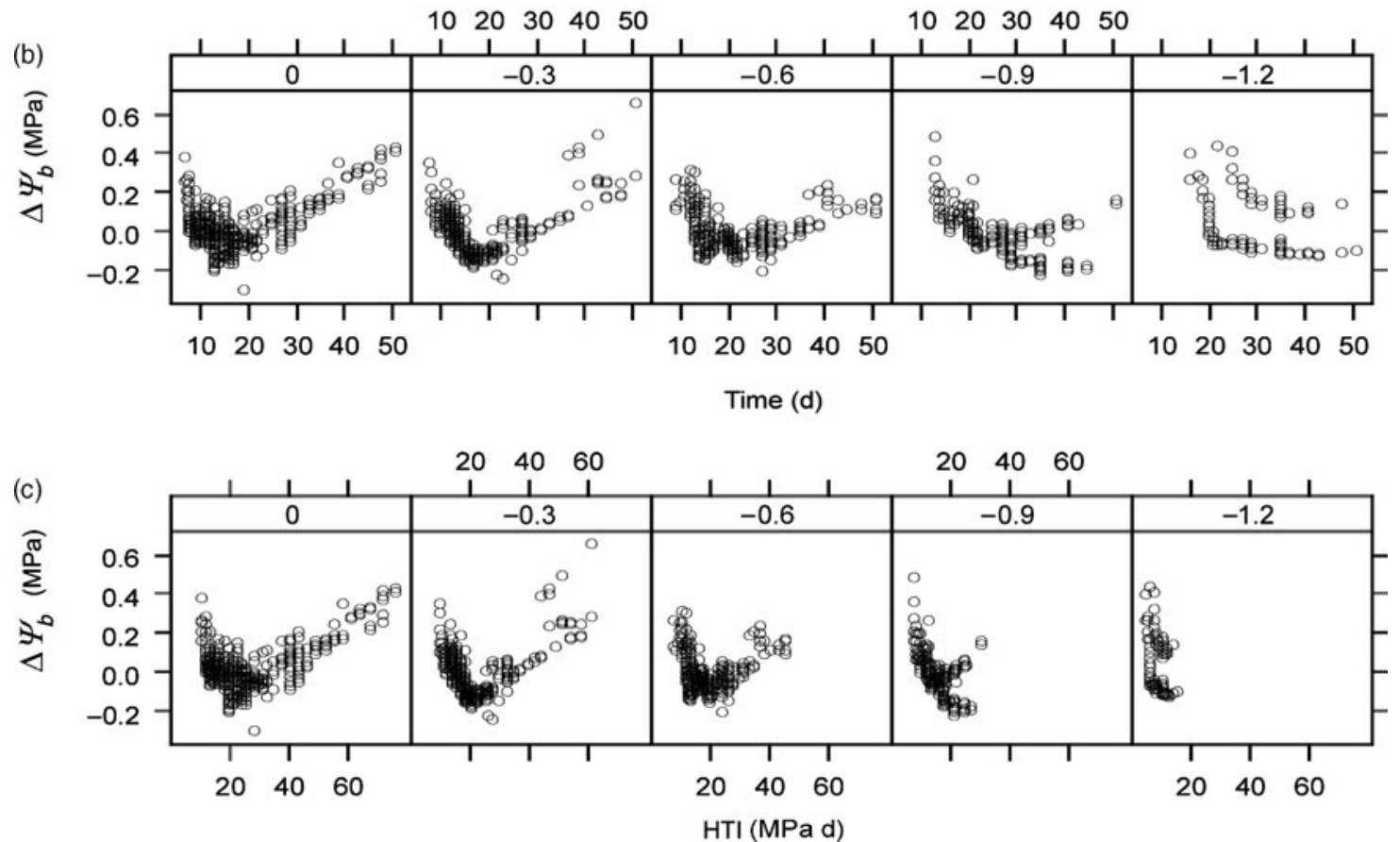

Figure 2. $\Delta \Psi_{b}$ plotted against (a) seed percentile $(\mathrm{G})$; (b) time to germination; and (c) hydro-time index (HTI). Data are plotted for $\Psi$ treatments (L to R): $0,-0.3,-0.6,-0.9$ and $-1.2 \mathrm{MPa}$. Data for each $\Psi$ treatment include combinations with all suboptimal temperature treatments.

were fitted with an additional term for the increase of $\Psi_{b}(\mathrm{G})^{\prime}$ with time to germination, as follows:

Model 2

$$
\Psi_{b}(\mathrm{G})^{\prime}=\Psi_{b}(\mathrm{G})_{\text {pred }}+k\{t(\mathrm{G})-25\} \text { for } t(\mathrm{G})>25 \mathrm{~d}
$$

Model 3

$$
\begin{gathered}
\Psi_{b}(\mathrm{G})^{\prime}=\Psi_{b}(\mathrm{G})_{\text {pred }}+k^{\prime}\{[(\Psi+1.5) t(\mathrm{G})]-20\} \\
\text { for }[(\Psi+1.5) t(\mathrm{G})]>20 \mathrm{MPad}
\end{gathered}
$$

where $k$ and $k^{\prime}$ are constants. Equation (7) adjusts $\Psi_{b}(\mathrm{G})$ as a function of time to germination, $t(\mathrm{G})$. Equation (8) accounts for the effect of water potential on $\Delta \Psi_{b}$ by adjusting $\Psi_{b}(\mathrm{G})$ as a function of a hydrotime index' to germination, $[(\Psi+1.5) t(\mathrm{G})]$. The base value for the 'hydro' component of the index is
$1.5 \mathrm{MPa}$. This is a fixed value chosen so that $(\Psi+1.5)$ is positive for all water potential treatments used in this study. For dry conditions (e.g. $-1.2 \mathrm{MPa})$, the hydro-time index (HTI) and therefore $\Delta \Psi_{b}$ will be small; for moist conditions (e.g. $0 \mathrm{MPa}$ ), the HTI and therefore $\Delta \Psi_{b}$ will be large. Inspection of graphs of $\Delta \Psi_{b}$ versus HTI revealed that the linear increase in $\Delta \Psi_{b}$ occurs from HTI $\approx 20 \mathrm{MPa}$ d onwards (Fig. $2 \mathrm{c}$ ).

The alternative hydrothermal time models [Model 1, without adjustment to $\Psi_{b}(\mathrm{G})$, or Models 2 and 3, with adjustments to $\Psi_{b}(\mathrm{G})$, as specified in equations (7) and (8)] were compared for their likelihood using the Akaike Information Criterion (AIC). The AIC is a penalized log-likelihood criterion, i.e. it calculates the likelihoods of alternative models fitted to the same data, but reduces the likelihood of each model in proportion to the number of parameters that it uses (Akaike, 1974; Burnham and Anderson, 2001). 
The lower (more negative) the AIC value, the greater the likelihood of the model.

The AIC values (Table 1) suggest that Model 3 [equation (8)], which adjusts $\Psi_{b}(\mathrm{G})$ as a function of HTI, gives the best fit to the measurement data. Model 2 [equation (7)] is also superior to Model 1. Models 2 and 3 also have the advantage compared with Model 1 of offering a mechanistic explanation of departures from normality in the frequency distribution for $\Psi_{b}(\mathrm{G})$, i.e. an initial normal distribution is skewed by increases in the $\Psi_{b}(\mathrm{G})$ values for the slower-germinating seeds, which occur as the seed proceeds to germination.

Figures $3 \mathrm{a}, \mathrm{c}$ and e show predicted germination time courses at $\Psi=0 \mathrm{MPa}$ for Models 1, 2 and 3, compared with actual germination data. These clearly show that the model with the HTI-adjusted seed base water potential (Model 3) more correctly predicts germination at all temperatures, whereas the unadjusted model (Model 1) incorrectly predicts higher germination percentages than actually occurred in most cases. Model 2 makes generally correct predictions but incorrectly predicts germination at $12.5^{\circ} \mathrm{C} /$ $0 \mathrm{MPa}$. The comparisons between models shown in Fig. 3 were consistent with results at other water potentials, as shown by the plots of model residuals (observed values minus predicted values) for germination percentiles of all suboptimal data (Fig. 3b, d and f). In particular, Fig. $3 b$ shows a pronounced curvature in the distribution of residuals for Model 1 when plotted against predicted germination percentile.

\section{Hydrothermal models at supra-optimal temperatures}

Raw residuals $\left[\Delta \Psi_{b}\right.$, calculated from equations (4) and (5)] were calculated for the germination data at supra-optimal temperatures. Residuals were used to test the RFS model assumption that thermal time accumulation continues to increase linearly above $T_{d}$. Inspection of Fig. 1a suggests that $T_{d}=20^{\circ} \mathrm{C}$. This was the value used in subsequent analysis. Figure $4 \mathrm{a}$ shows $\Delta \Psi_{b}$ at supra-optimal temperatures, plotted against temperature. In Fig. $4 \mathrm{a}, \Delta \Psi_{b}$ increased in magnitude with increasing temperatures above $20^{\circ} \mathrm{C}$ as predicted by the RFS model. Equation (6) was therefore fitted to the data using least squares regression. The estimated RFS model was $\Delta \Psi_{b}=0.0729\left(T-T_{d}\right)$ where $T_{d}=20^{\circ} \mathrm{C}$, where $T_{d}=$ $20^{\circ} \mathrm{C}$. However, Fig. 4 a also shows that actual $\Delta \Psi_{b}$ values varied widely around the estimated model at all temperature levels. It appears that temperature did not fully account for variation in $\Delta \Psi_{b}$ at supra-optimal temperature. For suboptimal temperatures, $\Delta \Psi_{b}$ increased as seeds proceeded towards germination. A similar increase may occur at supra-optimal temperatures. Figure $4 \mathrm{~b}$ shows $\Delta \Psi_{b}$ versus time to germination, for each supra-optimal temperature. This shows that $\Delta \Psi_{b}$ did increase non-linearly with time to germination but the form of the relationship varied with temperature. To account for this, $\Delta \Psi_{b}$ was plotted against thermal time accumulated above $T_{d}$, i.e. $[T-20] t(\mathrm{G})$, for each level of water potential in the experiment (Fig. 4c). Doing this revealed that $\Delta \Psi_{b}$ was strongly correlated with thermal time, although the form of the relationship varied between water potential treatments. This variation between water potential treatments followed a consistent trend, with $\Delta \Psi_{b}$ increasing at a slower rate per unit of thermal time as water potential became drier. Therefore $\Delta \Psi_{b}$ was also plotted against a 'supra-optimal hydrothermal time index' $\left[\right.$ SOHTI $\left.=(\Psi+1.5)\left(T-T_{d}\right) t(G)\right]$. Note that this supra-optimal hydrothermal time index (SOHTI) differs from the population hydrothermal time constant $\theta_{\mathrm{HT}}$, because it is a variable, not a model parameter. It is calculated from a base temperature of $T_{d}=20^{\circ} \mathrm{C}$ rather than $T_{b}$, and from the time to germination $t(G)$, which varies for each seed. The base water potential (1.5 MPa) used to calculate the SOHTI is a fixed value, arbitrarily chosen so that $(\Psi+1.5)$ is positive for all water potential treatments used in this study. This arbitrary value was used because attempts to analytically derive a base water potential for SOHTI did not produce a convergent solution. The relationship between $\Delta \Psi_{b}$ and SOHTI was quite consistent for all data, and appeared to have an asymptotic exponential form with an asymptote of $1.5 \mathrm{MPa}$ (Fig. $4 \mathrm{~d}$ ). This suggests that the temperaturedriven shift in $\Psi_{b}(\mathrm{G})$ proposed by Alvarado and Bradford (2002) and by Rowse and Finch-Savage (2003) was (1) not instantaneous but occurred over time; and (2) was also influenced by the water potential of the seed's environment.

A regression was fitted to the relationship between $\Delta \Psi_{b}$ and SOHTI using an asymptotic exponential equation (Crawley, 2002). The model was fitted using the ' $n l s$ ' function in program $\mathrm{R}$ ( $\mathrm{R}$ Development Core Team, 2007). This function simultaneously estimated all model parameters using an iterative least-squares procedure. The estimated model was $\Delta \Psi_{b}=1.448-\{1.562 \exp [-0.00596(\mathrm{SOHTI})]\}$, therefore the following equation was used to adjust seed $\Psi_{b}(\mathrm{G})$ [equation (4)] as a function of SOHTI:

$$
\begin{aligned}
\Psi_{b}(\mathrm{G})^{\prime}= & \Psi_{b}(\mathrm{G})_{\text {pred }}+1.448 \\
& -\{1.562 \exp [-0.00596(\mathrm{SOHTI})]\}
\end{aligned}
$$

The adjusted $\Psi_{b}(\mathrm{G})^{\prime}$ was then used as an input into a hydrothermal time model, with other model parameters derived from Model 3 for suboptimal temperatures, i.e. $\theta_{H T}=176 \mathrm{MPa}{ }^{\circ} \mathrm{Cd}, T_{b}=9.0^{\circ} \mathrm{C}$. Figures $5 \mathrm{a}$ and $\mathrm{c}$ show predicted germination time 


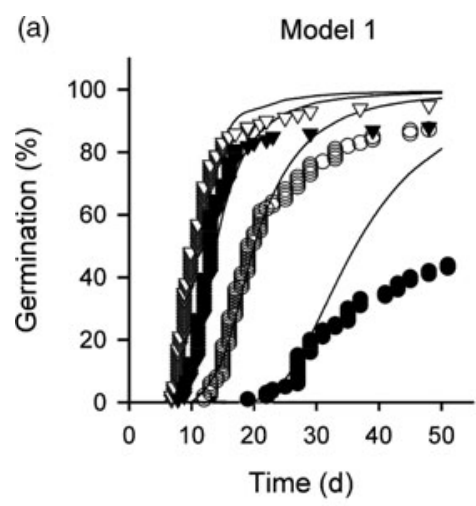

(b)

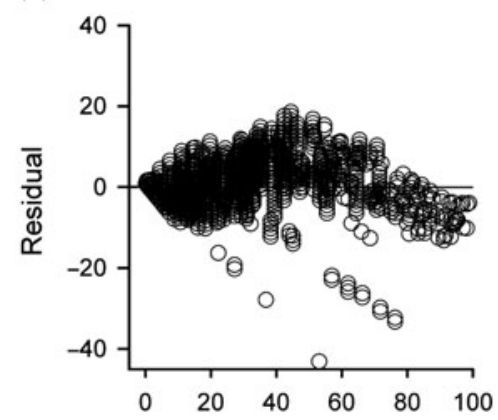

Predicted seed percentile $(G)$
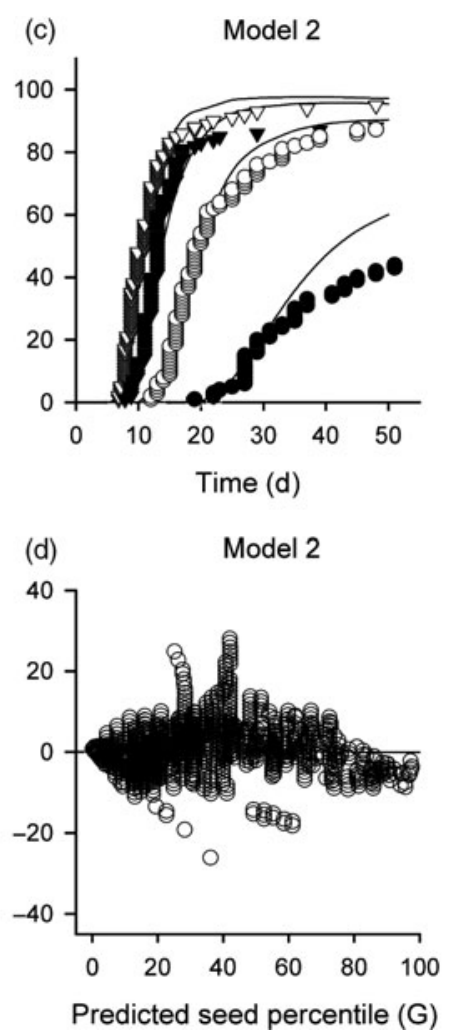

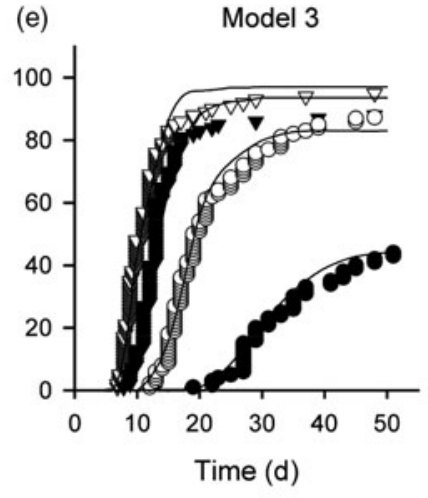

(f) Model 3

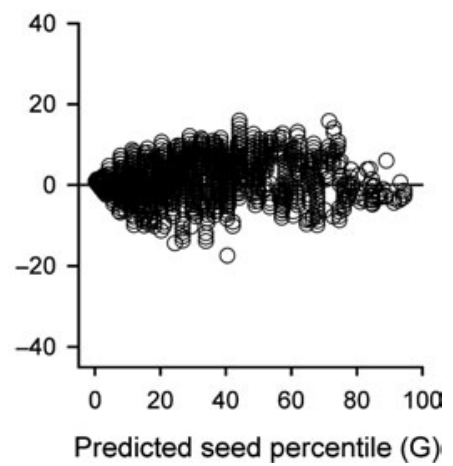

Figure 3. Comparison of Models 1, 2 and 3 for suboptimal germination data. (a), (c) and (e) Predicted and actual germination time course for Models 1, 2 and 3 at $\Psi=0 \mathrm{MPa}$. Data are plotted for each temperature as follows: $\bullet=12.5^{\circ} \mathrm{C}, \mathrm{O}^{\circ}=15^{\circ} \mathrm{C}$, $\boldsymbol{\nabla}=17.5^{\circ} \mathrm{C}, \nabla=20^{\circ} \mathrm{C}$. Model predictions are shown by the solid lines. (b), (d) and (f) Residuals [observed minus predicted seed percentile $(G)$ ] versus predicted seed percentiles for all suboptimal germination data.

courses at $\Psi=0 \mathrm{MPa}$ for the RFS and SOHTI models compared with actual germination data. The RFS model correctly predicted germination up to $10 \mathrm{~d}$, but markedly overpredicted germination from $10 \mathrm{~d}$ onwards (Fig. 5a). In contrast, the SOHTI model more accurately predicted reduced germination at all supra-optimal temperatures (Fig. 5c). The comparisons between models at $\Psi=0$ (Fig. 5a and c) were consistent with results at other water potentials, as shown by: (1) AIC values (Akaike, 1974) of 81.8 and -744 for the RFS and SOHTI models, respectively; and (2) plots of model residuals for germination percentiles of all supra-optimal data (Fig. $5 b$ and d). Figure $5 b$ shows how the residuals for the RFS model were strongly biased when plotted against predicted germination percentile. Figure $5 \mathrm{~d}$ shows how this bias was corrected using the SOHTI model.

\section{Ungerminated seeds}

For treatments at low temperatures $\left(12.5\right.$ or $\left.15^{\circ} \mathrm{C}\right)$ and for all supra-optimal temperatures, the higher seed percentiles did not germinate. The unadjusted hydrothermal model [equation (1)] predicted that most of these seeds should have germinated, because they should have accumulated sufficient hydrothermal time. In contrast, the hydrothermal models using adjusted seed base water potentials [equations (7), (8), (9)] correctly predicted no germination by the higher seed percentiles, because they adjusted seed base water potential upwards, as described in the previous section. This shift in base water potential towards zero resulted in the high percentile seeds failing to germinate because they accumulated insufficient hydrothermal time to germinate, even under moist conditions. An alternative explanation for this lack of germination might be the induction of secondary dormancy at both suboptimal and supra-optimal temperatures. The extent of dormancy in all remaining seeds was tested after $50 \mathrm{~d}$ by placing the seeds in germination trays with moist germination substrate $\left(\Psi_{b}=0 \mathrm{MPa}\right)$ and incubating at optimum temperature $\left(20^{\circ} \mathrm{C}\right)$ for a further $75 \mathrm{~d}$. For ungerminated seed from the suboptimal $T$ and $\Psi$ treatments, $>90 \%$ germinated within $20 \mathrm{~d}$ of being placed in optimal conditions. Seeds from the driest treatments $(-1.2$ and $-0.9 \mathrm{MPa})$ were slower to respond, typically requiring more than $30 \mathrm{~d}$ to reach $>90 \%$ germination. This suggests that the lack of germination during the experimental period of $50 \mathrm{~d}$ was due to insufficient hydrothermal time being accumulated by the seeds. For temperature treatments 

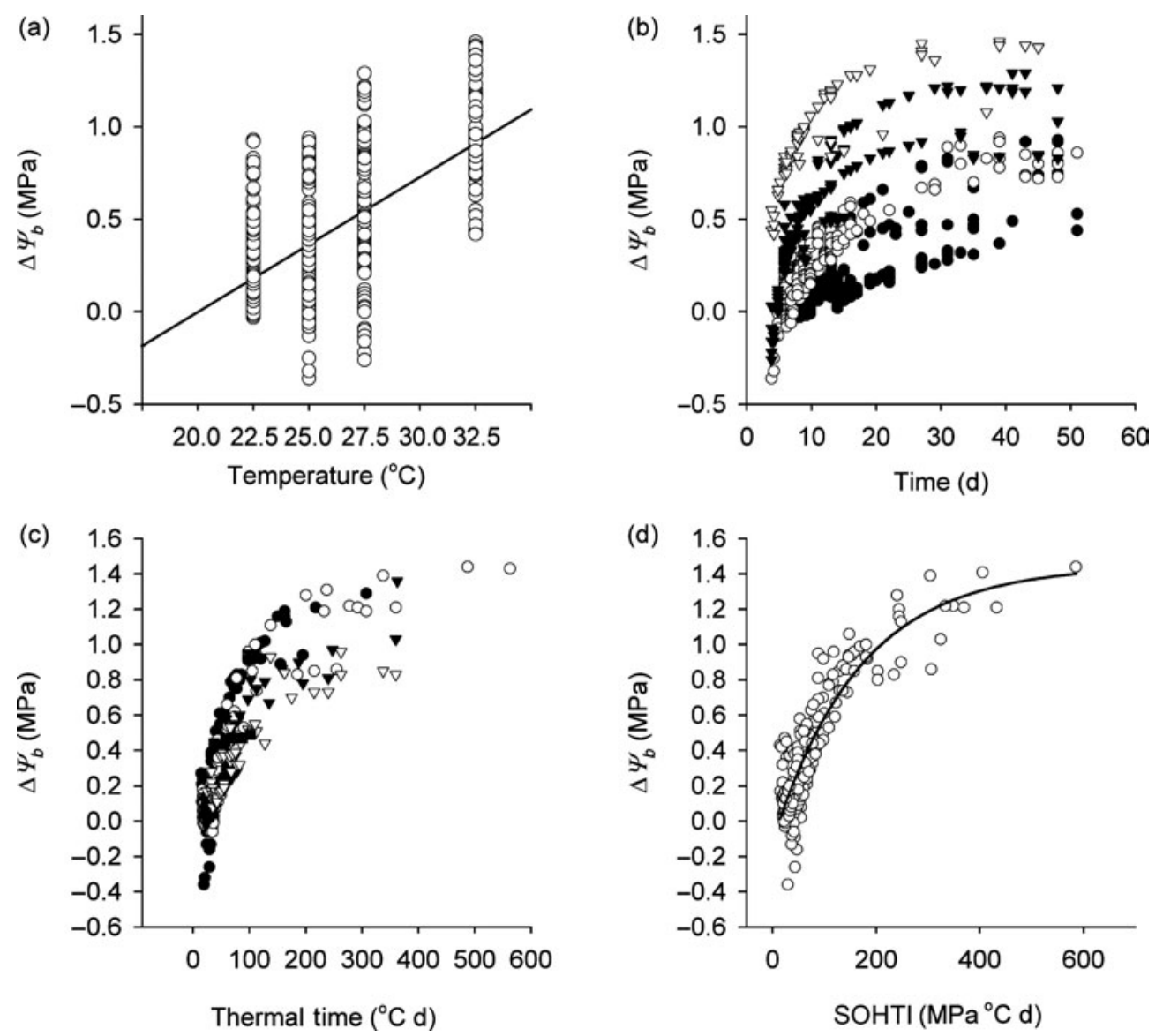

Figure 4. $\Delta \Psi_{b}$ plotted against (a) temperature. The solid line is $\Delta \Psi_{b}=0.0729(T-20)$, where $T=$ temperature; (b) time to germination for each temperature level $\left(-22.5^{\circ} \mathrm{C}, \mathrm{O}=25^{\circ} \mathrm{C}, \boldsymbol{\nabla}=27.5^{\circ} \mathrm{C}, \nabla=32.5^{\circ} \mathrm{C}\right)$; (c) thermal time to germination $(T-20) t(\mathrm{G})$ for each water potential $(\bullet=0 \mathrm{MPa}, \mathrm{O}=-0.3 \mathrm{MPa}, \boldsymbol{\nabla}=-0.6 \mathrm{MPa}, \nabla=-0.9 \mathrm{MPa}, \mathbf{\square}=-1.2 \mathrm{MPa})$; $(\mathrm{d})$ supraoptimal hydrothermal time index (SOHTI). The solid line is $\Delta \Psi_{b}=1.448-\{1.562 \exp [-0.00596(\mathrm{SOHTI})]\}$.

of $27.5^{\circ} \mathrm{C}$ and $32.5^{\circ} \mathrm{C}$, and for all supra-optimal temperature treatments where $\Psi$ was $\leq-0.6 \mathrm{MPa}$, seed germination during the $50 \mathrm{~d}$ incubation period was markedly reduced (Fig. 5). After a further $75 \mathrm{~d}$ under optimum conditions, seeds from treatments where $\Psi \geq-0.6 \mathrm{MPa}$ achieved $\sim 80 \%$ germination, except for the $32.5^{\circ} \mathrm{C}$ treatments. Towards the end of the $75 \mathrm{~d}$, ungerminated seeds began to decompose, especially seeds from $27.5^{\circ} \mathrm{C}$ and $32.5^{\circ} \mathrm{C}$ treatments.

\section{Discussion}

\section{Implications for models of germination at suboptimal temperatures}

At suboptimal temperatures, a conventional hydrothermal model [equation (1)] gave a reasonable fit to the germination data, but overpredicted germination rates for slower-germinating seeds and consequently overpredicted germination percentages. Modified models which adjusted $\Psi_{b}(\mathrm{G})$ upward as a linear function of time or hydro-time index (HTI) to germination were markedly more accurate for all combinations of $\Psi$ and suboptimal Tused in this study. While departures of actual seed behaviour at suboptimal temperatures from the conventional hydrothermal model are frequently reported in the literature, the hypothesis that these departures are caused by $\Psi_{b}(\mathrm{G})$ varying as a function of germination time course has not been widely tested. Allen et al. (2000) reported that for a range of dry climate plant species, $\Psi_{b}(50)$ of seeds appears to increase towards zero when incubated at temperatures close to $T_{b}$. This behaviour was successfully modelled by allowing $\Psi_{b}(50)$ to increase under these conditions, although the authors do not describe the methods used to calculate that increase.

A more detailed paper by Gianinetti and Cohn (2007) describes the induction of secondary dormancy in red rice (Oryza sativa L.) in terms of an upwards shift in $\Psi_{b}(\mathrm{G})$. This upwards shift was modelled as a function of hydro-time accumulated during germination, an approach equivalent to Model 3 in this study. More commonly, departures from the conventional hydrothermal model are achieved by specifying 

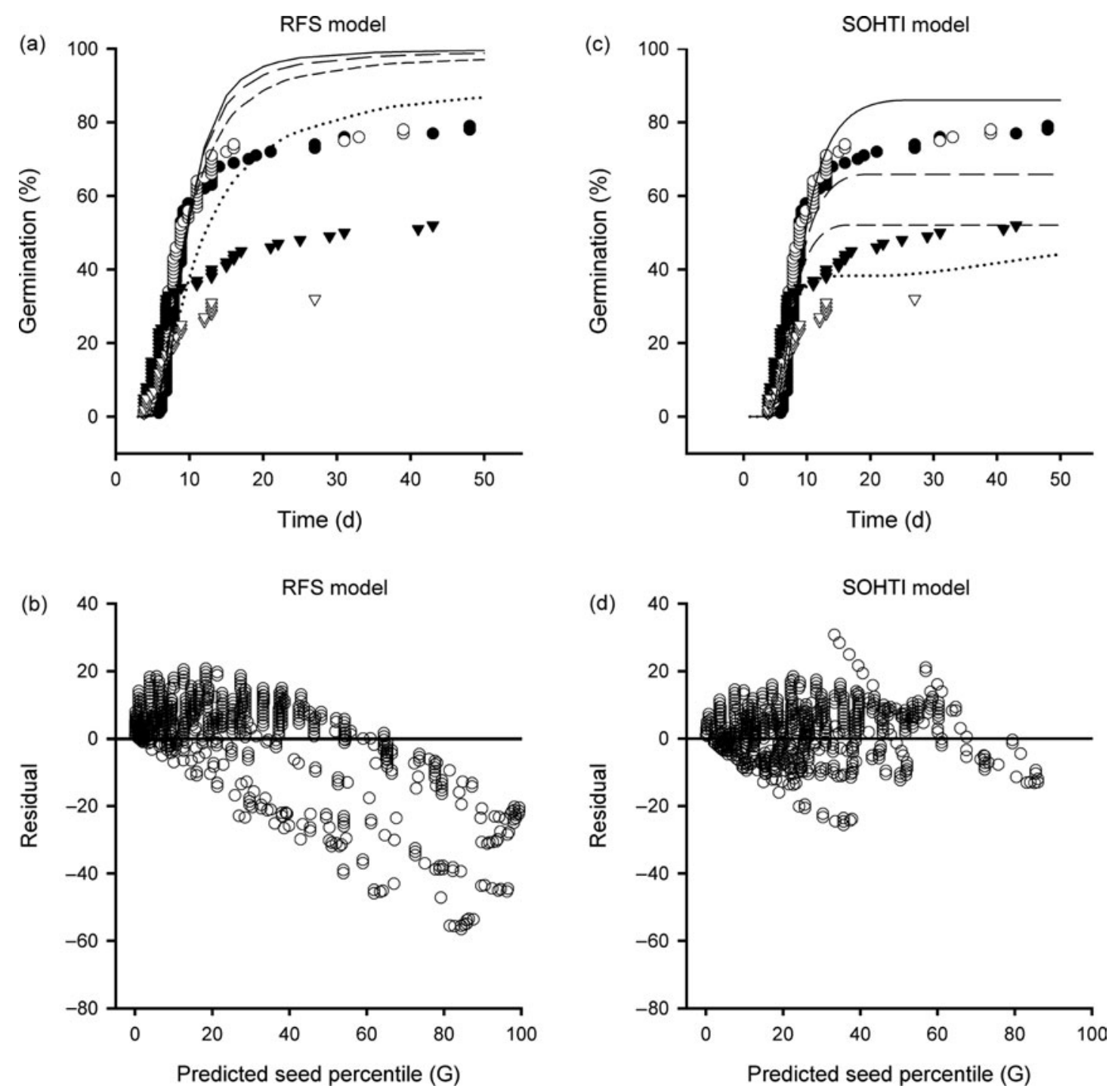

Figure 5. Comparison of the RFS with the SOHTI model. (a) and (c) Predicted and actual germination time course for $\Psi=0 \mathrm{MPa}$. Data are plotted for each temperature as follows: $0=22.5^{\circ} \mathrm{C}, \mathrm{O}=25^{\circ} \mathrm{C}, \boldsymbol{\nabla}=27.5^{\circ} \mathrm{C}, \nabla=32.5^{\circ} \mathrm{C}$. Model predictions are plotted as $-\longrightarrow=22.5^{\circ} \mathrm{C},---=25^{\circ} \mathrm{C},-----=27.5^{\circ} \mathrm{C}, \ldots \ldots \ldots .+32.5^{\circ} \mathrm{C}$. (b) and (d) Residuals [observed minus predicted seed percentile $(\mathrm{G})$ ] versus predicted seed percentiles for all supra-optimal germination data.

separate models for different levels of ambient $T$ and $\Psi$. Ni and Bradford (1992) and Dahal and Bradford (1994) reported a downwards adjustment in $\Psi_{b}(\mathrm{G})$ for tomato (Lycopersicon aesculentum Mill.) seeds incubated under low water potentials, so that seeds became more germinable under these conditions. Dahal and Bradford (1994) accounted for this adjustment by fitting two separate hydrothermal models to the data, one for germination data where $\Psi$ was drier than $-0.5 \mathrm{MPa}$ [with a lower mean $\Psi_{b}(\mathrm{G})$ ] and one for data when $\Psi$ was moister than $-0.5 \mathrm{MPa}$ [with a higher mean $\left.\Psi_{b}(\mathrm{G})\right]$.

Kebreab and Murdoch (1999) described results for germinating broomrape (Orobanche aegyptiaca Persoon), where: (1) $T_{b}$ apparently varied with ambient water potential, in contrast to the conventional hydrothermal model assumption of a single $T_{b}$ value for the whole seed population; and (2) the mean base water potential $\Psi_{b}(50)$ was found to vary with temperature, being $-2 \mathrm{MPa}$ between 14 and $23^{\circ} \mathrm{C}$ but increasing towards zero at both higher and lower temperatures. These variations in $T_{b}$ and $\Psi_{b}$ for $O$. aegyptiaca were modelled by the authors as functions of ambient water potential. However, neither Dahal and Bradford (1994) nor Kebreab and Murdoch (1999) consider the effect of the longer germination times that occur when ambient $T$ and $\Psi$ are not optimal. One may speculate, for example, that apparent shifts in $\Psi_{b}(50)$ with low temperature described by Kebreab and Murdoch (1999) arise because of slower germination at these temperatures. Similarly, the shifts in $\Psi_{b}(\mathrm{G})$ described by Dahal and Bradford (1994) for tomato at 
low water potentials also occurred at low temperatures, suggesting that slower germination times may have been a factor in causing these shifts.

\section{Implications for models of germination at supra- optimal temperatures}

At suboptimal temperatures, imbibed radiata pine seeds whose $\Psi_{b}(\mathrm{G})$ distribution shifts upwards because conditions do not allow rapid germination, may remain ungerminated until they experience sustained optimal conditions. This seems to be a useful 'bet-hedging' adaptation for radiata pine seeds which are otherwise fully germinable when mature (Kao and Rowan, 1970; Rimbawanto et al., 1988).

However, at supra-optimal temperatures, the adaptive value of reduced germination under moist conditions is less clear, because germination could proceed rapidly under these conditions. In this study, plots of GR versus $T$ indicated that radiata pine seeds can accumulate thermal time linearly up to $27.5^{\circ} \mathrm{C}$ (Fig. 1a), suggesting that seeds would be able to germinate very rapidly at this temperature, in the absence of an upwards adjustment in seed base water potential caused by temperatures where $T>T_{d}$. Furthermore, after germination young radiata pine seedlings can grow successfully under moist conditions with daytime temperatures $\approx 30^{\circ} \mathrm{C}$ (Hellmers and Rook, 1975). More generally, Bradford (2002) comments that the reasons for the decrease in seed GR and inhibition of germination at supra-optimal temperatures are not obvious, and that the most commonly offered explanation, i.e. non-linearity in thermal time accumulation due to denaturation of proteins, is unlikely to occur at temperatures as low as $20^{\circ} \mathrm{C}$.

A possible explanation is offered by the model for germination at supra-optimal temperatures proposed in this paper [equation (9)]. In contrast to the RFS and $\mathrm{AB}$ models, which explain reduced germination solely in terms of supra-optimal temperatures, equation (9) shows that reduced germination occurs as an asymptotic function of time and is also dependent on ambient $\Psi$, with the rate of reduction occurring fastest under moist conditions. It seems counter-intuitive that seeds would most rapidly inhibit their germination when ambient moisture is ideal for germination and seedling growth. However, it makes more sense if the upwards adjustment in seed base water potential is an adaptation to cope with a 'false break', a feature of mediterranean and other summer-dry climates where unseasonal late summer rains may trigger seed germination but germinated seedlings subsequently die because the rains do not persist (Norman et al., 1998). Radiata pine originates from the 'fog belt' along the coast of central California, where the climate is classified as cool mediterranean (Dallman, 1998) and summer droughts are the norm. One may speculate that radiata pine seeds that imbibe and proceed towards germination during a 'false break' in late summer (1) will not germinate due to a rapid upwards shift in seed base water potential caused by supraoptimal temperatures combined with moist soils; but (2) will germinate once autumn rains bring cooler temperatures, when seedling survival is more likely. This rapid shift in seed base water potential is not needed if the 'false break' does not occur, even though soil temperatures will be supra-optimal much of the time, because ambient soil moisture potentials will be drier than the unadjusted seed base water potentials and the seeds will not germinate anyway.

However, this speculation must be qualified by saying that the time-, temperature- and moisturedependent adjustments to seed base water potential reported here would need to be observed under field conditions similar to the natural habitat of radiata pine on the Central Californian coast - if only to verify that they are a real feature of the seed ecology of radiata pine, and not an artefact of the constant incubation conditions used in this study.

\section{Acknowledgements}

Lissy Kajer, Keith Pollock, Stephen Stilwell and Rosemary Weaver provided technical assistance with the germinations. Dr Bill Finch-Savage and Kath Phelps (WHRI, University of Warwick) provided comments and advice at various times during this study. The manuscript also benefited from the comments of two anonymous reviewers.

\section{References}

Akaike, H. (1974) A new look at the statistical model identification. IEEE Transactions on Automatic Control AC19, 716-723.

Allen, P.S., Meyer, S.E. and Khan, M.A. (2000) Hydrothermal time as a tool in comparative germination studies. pp. 401-410 in Black, M.; Bradford, K.J.; Vasquez-Ramos, J. (Eds) Seed biology: advances and applications. Oxford, CAB International.

Alvarado, V. and Bradford, K.J. (2002) A hydrothermal time model explains the cardinal temperatures for seed germination. Plant, Cell and Environment 25, 1061-1069.

Bradford, K.J. (2002) Applications of hydrothermal time to quantifying and modelling seed germination and dormancy. Weed Science 50, 248-260.

Burnham, K.P. and Anderson, D.R. (2001) Kullback-Leibler information as a basis for strong inference in information studies. Wildlife Research 28, 111-119.

Crawley, M.J. (2002) Statistical computing: an introduction to data analysis using S-Plus. Chichester, UK, Wiley.

Dahal, P. and Bradford, K.J. (1994) Hydrothermal time analysis of tomato seed germination at suboptimal temperature and reduced water potential. Seed Science 4, 71-80. 
Dallman, P.R. (1998) Plant life in the world's mediterranean climates: California, Chile, South Africa, Australia, and the Mediterranean Basin. Berkeley, California, University of California Press.

Finch-Savage, W.E. (2004) The use of population-based threshold models to describe and predict the effects of seedbed environment on germination and seedling emergence of crops. pp. 51-95 in Benech-Arnold, R.L.; Sanchez, R.A. (Eds) Handbook of seed physiology: applications to agriculture. Binghamton, New York, Haworth Reference Press.

Gianinetti, A. and Cohn, M.A. (2007) Seed dormancy in red rice. XII: Population-based analysis of dry-afterripening with a hydrotime model. Seed Science Research 17, 253-271.

Grundy, A.C., Phelps, K., Reader, R.J. and Burston, S. (2000) Modelling the germination of Stellaria media using the concept of hydrothermal time. New Phytologist 148, 433-444.

Gummerson, R.J. (1986) The effect of constant temperatures and osmotic potentials on germination of sugar beet. Journal of Experimental Botany 37, 729-741.

Hardegree, S.P. and Emmerich, W.E. (1990) Effect of polyethylene glycol exclusion on the water potential of solution-saturated filter paper. Plant Physiology 92, 462-466.

Hellmers, H. and Rook, D.A. (1975) Air temperature and growth of radiata pine seedlings. New Zealand Journal of Forest Science 3, 271-285.

ISTA (2003) International rules for seed testing. Adopted at the extraordinary meeting 2002, Santa Cruz, Bolivia to become effective on 1st January 2003. Bassersdorf, Switzerland, International Seed Testing Association.

Kao, C. and Rowan, K.S. (1970) Biochemical changes in seed of Pinus radiata during stratification. Journal of Experimental Botany 21, 869-873.
Kebreab, E. and Murdoch, A.J. (1999) Modelling the effects of water stress and temperature on germination rate of Orobanche aegyptiaca seeds. Journal of Experimental Botany $334,655-664$.

Ni, B.-R. and Bradford, K.J. (1992) Quantitative models characterizing seed germination responses to abscisic acid and osmoticum. Plant Physiology 98, 1057-1068.

Norman, H.C., Cocks, P.S., Smith, F.P. and Nutt, B.J. (1998) Reproductive strategies in Mediterranenan annual clovers: germination and hardseededness. Australian Journal of Agricultural Research 49, 973-982.

Phelps, K. and Finch-Savage, W.E. (1997) A statistical perspective on threshold type germination models. pp. 361-368 in Ellis, R.H.; Black, M.; Murdoch, A.J.; Hong, T.D. (Eds) Basic and applied aspects of seed biology. Dordrecht, Kluwer.

R Development Core Team (2007) R: A language and environment for statistical computing. Vienna, Austria, R Foundation for Statistical Computing.

Rimbawanto, A., Coolbear, P. and Firth, A. (1988) Artificial ripening of prematurely harvested cones of New Zealand Pinus radiata and its effect on seed quality. New Zealand Journal of Forestry Science 18, 149-160.

Rowse, H.R. and Finch-Savage, W.E. (2003) Hydrothermal threshold models can describe the germination response of carrot (Daucus carota) and onion (Allium cepa) seed populations across both sub- and supra-optimal temperatures. New Phytologist 158, 101-108.

Received 24 October 2008

accepted after revision 27 February 2009 (C) 2009 Cambridge University Press 